\title{
EDITORIAL
}

\section{Developmental psychiatry grown up}

\author{
Jonathan Green
}

Child mental health has developed two distinctive features in response to the challenges it has faced over time. The first is a focus on systems: the need to understand the psychopathology of children within a broad psychosocial context of family or community. The second is a developmental perspective, based on the fact that young patients are rapidly changing organisms.

The developmental approach to mental health has a long ancestry, but the past century or so has seen an extraordinarily dynamic branch of scientific progress, with an evolution of theory and a refinement of methodology, particularly in the increasing study of children themselves, rather than adult memories about childhood, and in prospective rather than retrospective study designs. Developmental psychopathology is the latest manifestation of this progression and one whose distinctive feature is the attempt to understand psychopathology in the light of normative biological and psychosocial development as well as abnormal influences (Cicchetti, 2001). Methodological rigour and scientific and analytic techniques have developed hugely - Jean Piaget built a magnificent theory largely out of scrupulous observations of just his own children, Sigmund Freud largely out of the same with his own patients and Mary Ainsworth from initial observations of 26 individuals. Some of the best current developmental studies have samples of hundreds and use increasingly sophisticated sampling, statistical analyses and experimental designs.

These studies have changed and continue to change our conceptualisation not only of childhood and childhood disorders but also of adult outcomes. For instance, many in the past would have associated developmental theory with the particular salience of early-life experience, but contemporary developmental psychopathology has intriduced theoretical models that acknowledge the importance of early development but emphasise strongly the ongoing and later effects of genetic releasing mechanisms, brain maturation and the moderating influence of later interplay between development and experience (Rutter, 2002). These ideas lead naturally to a life-span approach.

\section{Life-span psychiatry}

In this issue of $A P T$ we begin a new series of reviews of some of the current advances in this area. The specific focus is on significant recent advances in developmental neuropsychiatry relating to adult outcomes. The driver for this at least partly comes from the fact that a cohort of children that have grown up within modern child psychiatric practice are now reaching adulthood. And with them a number of disorders, recognised and investigated intensively over the past 30 years within child psychiatry, have as it were also grown up. Patients with these disorders, reaching adulthood, are now pressing for a continuation of services for their problems; and other adults reading about these problems are reconsidering themselves for the first time in their light.

It is not hard to see that this developmental perspective on adult functioning will grow apace in influence: certainly, patient demand is likely to increase in the medium term. Clinics for adult attention-deficit hyperactivity disorder (ADHD) and autistic-spectrum disorders are springing up around the world. The evolution in public attitudes in this area is striking. For instance, the notion of a genetic diathesis and clustering of familial traits in autism was sometimes an awkward subject to broach when I first began talking to parent groups in the late 1980s. Ten years later parents readily and often movingly began to describe their self-identified extended phenotypic traits. A paradigm of genetic and

Jonathan Green is a senior lecturer in child and adolescent psychiatry at the University of Manchester and an honorary consultant for Central Manchester and Manchester Children's Hospitals University NHS Trust (Academic Department of Child and Adolescent Psychiatry, Booth Hall Children's Hospital, Blackley, Manchester M9 7AA, UK. E-mail: jonathan.green@man.ac.uk). His main clinical and research interests are in social development and psychopathology, including pervasive developmental and attachment disorders, and in the study of the effectiveness and organisation of in-patient services. 
neurodevelopmental influences on development has achieved wide acceptance (sometimes excessively so, with an associated denial of psychosocial and interpersonal realities that is not supported by developmental psychopathology research).

Other trends also add to an increasing interest in the interplay between adult and child psychiatry at this time and make this series additionally timely. Adolescent and adult psychiatrists are increasingly focusing on issues of transition into adulthood and the provision of appropriate services for this, as evidenced by research into the early natural history of psychosis and the development of integrated early-onset psychosis services.

The series starts with two particular disorders that have 'grown up' in this way. In this issue Morris Zwi and Ann York discuss the notion of adult ADHD and in the next Tom Berney discusses adult manifestations of autistic-spectrum disorders (Berney, 2004). Topics for subsequent articles will include early developmental aspects of psychopathy and psychosis.

\section{Adult ADHD}

Zwi \& York (2004, this issue) illustrate a number of important facets of the general theme. There are interlinked methodological and conceptual issues about how to characterise adult ADHD and how to diagnose it. As these authors point out, one of the characteristics of disorders of this type - typical of developmental psychopathology - is that ADHD phenomena represent an exaggeration of normal developmental trait variation in activity levels, attentional control and impulsivity. Considerable research effort has gone into the investigation of the relationship between an ADHD diagnosis and trait variation in childhood. As Zwi \& York explain, these issues are still somewhat contentious. Will the understanding of the expression of ADHD in adulthood need to depend on similar normative studies of trait variation in adult life, or will extrapolation from longitudinal studies be sufficient? Zwi \& York note that there may be a distinction to be made here between adult ADHD in people diagnosed with the childhood disorder and people newly presenting with ADHD in adulthood. They make important related points about diagnostic criteria and prevalence estimation.

Asherson's commentary (2004, this issue) on Zwi $\&$ York's article provides a response from someone who has practical experience of running a service in the UK for adults with ADHD. Similar issues will arise in later articles in the series, reviewing the literature on Asperger syndrome and antisocial personality disorder.

We hope that this series will be of interest to adult psychiatry colleagues since there is likely to be increasing pressure on services to respond to the needs of this new group of 'grown-up' patients, rather different from regular clientele in many psychiatric clinics. If this does represent a real clinical development then there will no doubt be training implications, for both foundation and higher specialist training. In a future article in the series, Mary Eminson, who has been in the forefront of debating these issues in the Royal College of Psychiatrists, will outline the current state of play in this area.

\section{References}

Asherson, P. J. (2004) Bridging the service divide. Invited commentary on: Attention-deficit hyperactivity disorder in adults. Advances in Psychiatric Treatment, 10, 257-259.

Berney, T. (2004) Asperger syndrome from childhood to adulthood. Advances in Psychiatric Treatment, 10, in press.

Cicchetti, D. (2001) Reflections on the past and future of developmental psychopathology. In Research and Innovation on the Road to Modern Child Psychiatry. Volume 1 Festschrift for Professor Sir Michael Rutter (eds J. M. Green \& W. Yule), pp. 37-53. London: Gaskell Press.

Rutter, M. (2002) Development and psychopathology. In Child and Adolescent Psychiatry (4th edn) (eds M. Rutter \& E. Taylor), pp. 309-324. Oxford: Blackwell Press.

Zwi, M. \& York, A. (2004) Attention-deficit hyperactivity disorder in adults: validity unknown. Advances in Psychiatric Treatment, 10, 248-256. 